\title{
Psicologia Aplicada à Cardiologia: Um Estudo sobre Emoções Relatadas em Exame de Holter ${ }^{1}$
}

\author{
Ana Myriam Sánchez Bonomo \\ Tereza Cristina Cavalcanti Ferreira de Araujo ${ }^{2}$ \\ Universidade de Brasilia
}

\begin{abstract}
RESUMO - Estudos sobre fatores de risco associados a doenças do coração dão ênfase às emoções negativas e não esclarecem quanto aos efeitos cardíacos de vivências de alegria ou segurança. Realizou-se uma investigação com 30 pessoas submetidas ao exame de Holter 24 horas, visando identificar emoções e episódios de vida diária que ocorrem simultaneamente a arritmias cardíacas. Reuniram-se registros de arritmias, atividades e emoções, complementados por entrevistas semiestruturadas. Verificaram-se diferenças acentuadas entre gêneros: mulheres apresentaram mais arritmias que homens. Nas mulheres, arritmias ventriculares ocorreram mais simultaneamente à preocupação do que à ansiedade e não se mostraram relacionadas à raiva. A tristeza não foi associada a arritmias ventriculares por nenhum dos gêneros.
\end{abstract}

Palavras-chave: emoções; arritmias cardíacas; Holter; gênero.

\section{Psychology Applied to Cardiology: A Study on Emotions Reported during Holter Monitoring}

\begin{abstract}
Studies on risk factors associated with heart diseases emphasize the negative emotions and do not enlighten the cardiologic effects in response to joy or security. It was carried out an investigation with 30 people, submitted to 24 hours Holter monitoring, aiming at identifying emotions and episodes of daily life that occur simultaneously with cardiac arrhythmias. In doing so, records of arrhythmias, activities and emotions were gathered, additionally supported by semi-structured interviews. Great gender differences were found: women presented more arrhythmias than men. In women, ventricular arrhythmias occurred more simultaneously to worries than with respect to anxiety and were not related to anger. In both genders sadness was not associated with ventricular arrhythmias.
\end{abstract}

Keywords: emotions; cardiac arrhythmias; Holter; gender.

Inúmeros pesquisadores têm se esforçado para identificar fatores de risco para doenças do coração. Já foram reunidas evidências sobre determinantes biológicos, comportamentais e psicossociais, mas sabe-se, atualmente, que fatores orgânicos - como hipertensão, hipercolesterolemia, obesidade e diabetes - explicam apenas $40 \%$ das ocorrências de doenças cardíacas (Kubzansky \& Kawachi, 2000). Muitos estudos empíricos têm demonstrado a relação entre doenças cardiovasculares e fatores psicossociais, dentre os quais os mais investigados são: nível socioeconômico, suporte social, idade, gênero, padrão de comportamento tipo A, estresse, depressão e emoções negativas e positivas. Essa literatura especializada também aponta que a combinação de fatores psicossociais aumenta significativamente o risco potencial, além de atuar sinergicamente com fatores biológicos incrementando ainda mais a predisposição a eventos cardíacos

1 Trabalho desenvolvido pela primeira autora sob orientação da segunda durante o Curso de Pós-Graduação em Psicologia da Universidade de Brasília. Agradecimentos à Prof ${ }^{\mathrm{a}}$. Ângela Maria de Oliveira Almeida, da Universidade de Brasília, pelo auxílio técnico-científico na aplicação do software Alceste e à psicanalista Ana Velia Vélez de Sánchez Osella pela colaboração na análise das entrevistas.

2 Endereço para correspondência: . Instituto de Psicologia, Universidade de Brasília. Campus Darcy Ribeiro, Asa Norte. Brasília, DF. CEP 70910-900.E-mails: araujotc@unb.br; anamyriam@hotmail.com.
(Rozanski, Blumenthal \& Kaplan, 1999). Considerando o escopo da presente pesquisa, serão especialmente abordados os fatores gênero e emoção.

\section{Gênero e Predisposição para Doenças do Coração}

No Brasil, ser do gênero masculino é um dos fatores predisponentes para doenças do coração. Porém, a ocorrência de óbitos de origem cardíaca é maior entre mulheres, visto que, em 2000 , representou $31,30 \%$ do total de mortes, ao passo que este percentual para homens foi de 24,88\% (Ministério da Saúde, 2003). Para Frasure-Smith e Lesperance (1998), Kubzansky e Kawachi (2000) e Perez (2004), é preciso apontar que grande parte das pesquisas sobre predisposição é realizada com amostras compostas por homens, quando a taxa de mortalidade é maior entre mulheres.

Estudos a respeito da prevalência de doença coronária indicam que as taxas mais elevadas de mortalidade feminina estão associadas à presença de fatores hormonais, transmissão genética, diferenças de estrutura e funcionamento cerebral, assim como fatores psicossociais, tais como funcionamento psicológico, papel familiar e social (Perez, Nicolau, Romano \& Laranjeira, 2005). Ao comparar mulheres com e sem doenças cardiovasculares, durante o climatério, Favarato e 
Aldrighi (2001) obtiveram mais relatos de insatisfação com a vida, tristeza, choro fácil e nervosismo de mulheres coronariopatas do que de mulheres do grupo controle.

Diferenças de gênero na incidência de doença arterial coronariana e aterosclerose são também atribuídas aos efeitos do estrogênio. Mulheres pré-menopáusicas ou que fazem reposição hormonal apresentam proteção relativa para doenças coronarianas, derrame isquêmico e aterosclerose com relação aos homens de mesma idade, apresentando redução de 50\% na taxa de mortalidade (Lima \& Nussbacher, 1996). Porém, esta propalada "proteção feminina" merece ser melhor compreendida, pois aparentemente o que ocorre é que a curva de incidência dessas patologias em mulheres tem um atraso de aproximadamente 10 anos em relação à dos homens e acredita-se que, como a aterosclerose progride por anos, os eventos clínicos tiveram seu início anos antes (Perez, 2004; Rozanski \& cols., 1999).

A menopausa reflete-se na incidência de depressão, pois os estrogênios estão relacionados à metabolização de serotonina, de modo que sua presença teria um efeito semelhante ao de antidepressivos (Almeida \& Fráguas Jr., 1996). Na perimenopausa, período de quatro anos antes da instalação da menopausa, os níveis de estrogênio começam a oscilar, o que pode facilitar o aparecimento de sintomas depressivos (Favarato \& Aldrighi, 2001). Dados sugerem que o estresse psicossocial causa hipogonadismo hipotalâmico, ou seja, anormalidades ovarianas que reduzem os índices de estrogênio e cujas manifestações vão desde efeitos subclínicos até amenorréia, hipercolesterolemia e outros indicadores neuroendócrinos e comportamentais de estresse. A aterosclerose é então acelerada, predispondo à doença arterial coronariana e, possivelmente, ao derrame isquêmico (Rozanski \& cols., 1999). Pesquisas também mostram que o prognóstico de doenças coronárias é pior nas mulheres e que o gênero feminino está significativamente associado a complicações pós-operatórias em intervenções cirúrgicas cardíacas (Pinton, Carvalho, Miyazaki \& Godoy, 2006).

Até hoje, poucas investigações foram feitas sobre o efeito da hostilidade em mulheres. Em um trabalho pioneiro de avaliação do comportamento tipo A, não foi identificada associação entre esse comportamento e a incidência de doença coronariana em mulheres (Eaker, 1998). Os papéis sociais de gênero fazem com que as reações frente a uma mesma situação sejam diferentes. Homens tendem mais a expressar emoções negativas, vivenciando raiva com maior frequência, enquanto reprimem o medo, e expressando as emoções com maiores níveis de ativação fisiológica. As mulheres, por sua vez, relatam maior gama de emoções e as verbalizam mais intensamente, reprimindo tanto a experiência quanto a expressão da raiva. Ou seja, a supressão da raiva determinada pela socialização faz com que, nas mulheres, sentir ou demonstrar raiva gere outras emoções negativas como culpa, ansiedade, vergonha e depressão. Esse somatório de emoções aumenta a reatividade fisiológica, especialmente entre adolescentes e mulheres jovens, tendência que diminui com a idade, principalmente em episódios de raiva e de medo (Kubzansky \& Kawachi, 2000; Labouvie-Vief, Lumley, Jain \& Heinze, 2003; Lampert \& cols., 2002; Lavoiea, Miller, Conwaya \& Fleetb, 2001). Ao investigarem as diferenças da reatividade cardíaca frente a emoções induzidas por auto-relato de me- mórias em mais de 100 participantes, Labouvie-Vief e cols. (2003) constataram que a reatividade cardíaca diminuiu com a idade para ambos os gêneros, mas esse declínio foi muito mais acentuado entre as mulheres. Os efeitos da indução de tristeza e de alegria diminuíram com a idade para homens e mulheres, mas, para essas emoções, não houve diferenças significativas.

Percebe-se, então, que gênero é um fator que influencia as respostas cardíacas a determinadas emoções, mas não a outras.

\section{Emoções e suas Repercussões sobre o Ritmo Cardíaco}

$\mathrm{Na}$ literatura especializada, a descrição conceitual e teórica das emoções envolve diversas perspectivas, nem sempre consensuais ou convergentes. Entretanto, a maioria dos pesquisadores concorda que as emoções compreendem componentes afetivos, cognitivos e comportamentais, associados a componentes neurobiológicos. Ou seja, as emoções estimulam ações que determinam padrões de atividade fisiológica, os quais subsidiam o comportamento adaptativo. Elas integram o sistema neuro-anátomo-fisiológico e se originam na interação da pessoa com seu ambiente, regulando o comportamento diante das mudanças situacionais (Gallo \& Matthews, 2003; Kubzansky \& Kawachi, 2000). As emoções também são consideradas como processos constituídos subjetivamente, abrangendo dimensões biológicas, individuais, sociais e culturais (Neubern, 2000). Muitas vezes, é a interação social que determina quais emoções vão ocorrer, como serão expressas e quais consequências terão (Kubzansky \& Kawachi, 2000).

Considera-se que há, pelo menos, duas dimensões das emoções: intensidade e frequência. A intensidade refere-se à força das experiências emocionais e a frequência, à percentagem do tempo em que a emoção avaliada predomina sobre as demais. Vale salientar que as pessoas se lembram melhor da frequência de determinada emoção, o que as leva a superestimar a intensidade, tanto das emoções positivas quanto das negativas, mas, em especial, das negativas. Assim, situações com carga emocional seriam mais lembradas do que situações neutras (Thomas \& Diener, 1990).

Há grande variabilidade na expressão de emoções entre pessoas. Acredita-se que tanto a expressão exagerada quanto a inibição das emoções têm consequências para a saúde. Inibir a manifestação das emoções demanda esforço psicológico que, com o tempo, acarreta estresse cumulativo (Kubzansky \& Kawachi, 2000). Todavia, ao se estudar a relação entre a expressão e a inibição das emoções e incrementos da pressão arterial, em situações de role playing verificou-se que a expressão das emoções estava mais associada a aumentos da pressão arterial (Lipp, Pereira, Justo \& Matos, 2006). Mas, em outro trabalho, notou-se que quando o estilo de regulação é mais expressivo, a reatividade fisiológica é menor (Labouvie-Vief \& cols., 2003). Segundo alguns pesquisadores, a reatividade emocional que se desvia da norma, em ambas as direções, está relacionada com o risco de hipertensão e, provavelmente, com arritmias (Carels \& cols., 1999; Kubzansky \& Kawachi, 2000). 
Quanto à natureza, é possível agrupar as emoções em positivas e negativas, porém os pesquisadores ainda discordam se esses grupos são dois pólos de uma mesma dimensão, ou se formam dimensões distintas (Gallo \& Matthews, 2003; Richman \& cols., 2005). Reuniram-se evidências de associação entre doenças coronarianas e três emoções negativas específicas - ansiedade, raiva e depressão - mas, tendo em vista a diversidade de emoções existentes, é possível que outras categorias também estejam associadas, mas pouca atenção lhes foi dada até hoje (Kubzansky \& Kawachi, 2000).

Uma dificuldade suplementar no estabelecimento de vinculação entre reações orgânicas e emoções é que as emoções raramente ocorrem isoladas, sendo difícil discriminá-las. Além disso, sua nomeação exata constitui outro desafio, já que o ser humano é capaz de comunicar emoções sem adotar palavras. Portanto, as emoções podem ser mais ou menos diferenciadas, mesmo que o número de expressões verbais varie entre idiomas. Nesse sentido, pessoas de diferentes níveis educacionais vivenciam as mesmas emoções, mas podem identificá-las e nomeá-las diferentemente (Kubzansky \& Kawachi, 2000). Alguns estudiosos chegaram a definir seis categorias subordinadas à classificação geral de emoções negativas e positivas - raiva, medo, tristeza, alegria, amor e surpresa - e distribuíram os léxicos emocionais da língua inglesa em mais 20 subcategorias (Hupka, Lenton \& Hutchison, 1999). Essa diversidade de subcategorias dificulta sua nomeação precisa e, consequentemente, seu estudo.

\section{Emoções negativas}

Pesquisas mostraram que pacientes que apresentam isquemia ambulatorial durante avaliação de rotina têm maiores chances de haver vivenciado emoções negativas na hora anterior ao episódio isquêmico. Esses resultados sugerem que indivíduos com maior amplitude e frequência de reatividade emocional no cotidiano têm maiores chances de apresentar isquemia miocárdica (Carels \& cols., 1999). As emoções, principalmente as negativas, provocam alterações fisiológicas com impacto negativo no prognóstico da doença coronariana (Kubzansky \& Kawachi, 2000; Perez \& cols., 2005; Richman \& cols., 2005; Sirois \& Burg, 2003).

As emoções negativas podem ainda influenciar indiretamente tanto o risco quanto o prognóstico de pacientes com doença coronariana por influenciar a adesão a comportamentos prejudiciais à saúde como tabagismo, consumo de álcool e drogas, diminuição da qualidade do sono, má alimentação, obesidade e sedentarismo, além de seus efeitos sobre o suporte social e isolamento social (Kubzansky \& Kawachi, 2000; Sirois \& Burg, 2003). A isquemia miocárdica e o infarto do miocárdio podem ser provocados por fatores psicológicos, mas ainda não há evidências consistentes dessa associação em relação às arritmias cardíacas (Lampert \& cols., 2002), mesmo sabendo-se que "as emoções negativas como ansiedade e depressão podem afetar a estabilidade elétrica do coração através da alteração da regulação autonômica (especificamente, reduzindo a variabilidade da frequência cardíaca)" (Kubzansky \& Kawachi, 2000, p. 330) e que fatores comportamentais acarretam ativação simpática excessiva, desencadeando arritmias. É importante esclarecer que arritmias de origem comportamental são ocasionadas pela somatória de três condições: (a) instabilidade elétrica miocárdica, usualmente devido à doença coronariana prévia; (b) um evento desencadeador agudo, frequentemente relacionado a estresse mental; e (c) um estado psicológico crônico intenso, que muitas vezes inclui depressão e desesperança (Rozanski \& cols., 1999).

Inicialmente, não se conseguiu relacionar a ansiedade com as doenças cardíacas, porém estudos recentes que utilizaram o auto-relato de situações de ansiedade sugerem que a simples presença de sintomas de ansiedade pode ser um fator de risco, sem a necessidade de preencher critérios diagnósticos de estados patológicos (Burg, Lampert, Joska, Batsford \& Jain, 2004). A ansiedade mostra-se relacionada à ocorrência de eventos cardíacos ao afetar o controle cardíaco autonômico, podendo aumentar o risco de arritmias ventriculares fatais (Sirois \& Burg, 2003).

A manifestação de raiva e a hostilidade, enquanto traços de personalidade estáveis, está associada a maior isquemia, tanto durante testes de estresse mental quanto em situações cotidianas (Burg \& cols., 2004). Pacientes que reportaram raiva ou ansiedade moderada nos minutos anteriores a arritmias ventriculares sustentadas demonstraram maior pontuação nos testes de raiva-traço e ansiedade-traço, quando comparados a pacientes que não apresentaram arritmias relacionadas a emoções (Burg \& cols., 2004).

Raiva e ansiedade podem precipitar arritmias devido ao aumento da atividade simpática. A raiva não contribui somente para a aterogênese e o desenvolvimento de doenças cardíacas, mas também desencadeia eventos coronarianos agudos (Chang, Ford, Meoni, Wang \& Klag, 2002; Kubzansky \& Kawachi, 2000). Tanto a raiva, quanto os exercícios físicos podem causar infartos não fatais do miocárdio e/ou isquemia transitória (Lampert \& cols., 2002), porém, nas duas horas subsequentes a episódios de raiva, o risco relativo de infarto do miocárdio aumenta mais de duas vezes (Rozanski \& cols., 1999; Sirois \& Burg, 2003).

\section{Emoções positivas}

Conforme enfatizado anteriormente, o número de trabalhos sobre emoções positivas é escasso, quando comparado àqueles sobre emoções negativas. Cabe lembrar que taxonomias científicas sobre emoções básicas identificam apenas uma emoção positiva para cada três ou quatro emoções negativas, o que pode em parte explicar esta tendência (Fredrickson, 1998). Também há pouco consenso sobre o que pode ser considerado como uma emoção positiva. As teorias sobre emoção têm falhado ao tentar definir claramente as emoções positivas, que tendem a ser mais difusas do que as emoções negativas, mas considera-se que a presença de emoções positivas não consiste simplesmente na ausência de emoções negativas (Richman \& cols., 2005). Outro fator que certamente dificulta o estudo das emoções positivas é constatar que para emoções negativas específicas existem expressões faciais específicas, o que não ocorre com as emoções positivas, que compartilham entre si a expressão facial do sorriso. Do ponto de vista do funcionamento autonômico, algo semelhante acontece (Fredrickson, 1998). 
De fato, emoções positivas podem ter o potencial de reduzir efeitos do estresse sobre o sistema cardiovascular mesmo em face de eventos de vida negativos inevitáveis. Assim, otimismo e atitude positiva podem fazer com que os eventos negativos sejam encarados com a confiança de que o futuro reserva algo melhor. Nesse sentido, o estado emocional positivo gerado internamente modifica os efeitos adversos da exposição prolongada a emoções negativas (Danner, Snowden \& Friesen, 2001; Swan \& Carmelli, 1996). Em uma investigação com 1.041 homens entre 55 e 69 anos de idade, acompanhados por dois anos, verificou-se que para cada unidade de aumento da pontuação nos testes de curiosidade, ocorreu uma diminuição de $57 \%$ do risco de desenvolver hipertensão. Os efeitos da esperança e da curiosidade se mantiveram após o controle de variáveis como hábitos de saúde (Richman \& cols., 2005).

Em síntese, têm sido encontradas associações entre emoções e doenças. Isto é, as emoções podem influenciar o aparecimento de doenças específicas ou podem ser consequência de doenças, influenciando seu prognóstico. A relação entre emoções e a saúde cardiovascular é, então, bidirecional, tendo seu efeito cumulativo no decorrer do tempo (Kubzansky \& Kawachi, 2000). As pesquisas sobre emoções têm adotado, como procedimentos de coleta de dados, principalmente o auto-relato de emoções e diversas técnicas de indução em laboratório (Kubzansky \& Kawachi, 2000; Thomas \& Diener, 1990). É importante enfatizar que investigações experimentais restringem a apresentação de emoções àquelas mais reconhecidas. Por consequência, a identificação do papel de outras emoções na arritmogênese fica limitada.

Assim sendo, considera-se que o estudo de alterações emocionais que ocorrem no cotidiano de pacientes com monitorização eletrocardiográfica pode trazer à luz a participação de outras emoções no desencadeamento de reações cardiovasculares. Outra possibilidade interessante é identificar pacientes em risco de arritmias desencadeadas por emoções por meio de uma avaliação diagnóstica de rotina e relativamente simples, tal como o Exame de Holter 24 horas.

Então, visando tais interesses de natureza científica e clínica, foi realizada uma pesquisa a partir dos seguintes objetivos específicos: (a) identificar emoções que ocorram simultaneamente a arritmias em diferentes momentos do dia para um mesmo sujeito; (b) verificar a ocorrência de arritmias semelhantes em sujeitos com relatos emocionais semelhantes; e (c) descrever e analisar a associação entre emoções e atividades relatadas durante o Exame de Holter 24 horas (Sánchez, 2007).

\section{Método}

\section{Participantes}

A amostra foi constituída por 13 homens e 17 mulheres, com idade entre 48 e 69 anos, e com escolaridade mínima de ensino médio completo. Os participantes não faziam uso de medicação antiarrítmica, betabloqueadores ou antipsicóticos e tinham capacidade de comunicação oral e escrita preservada. Foram excluídos os pacientes que não preencheram esses critérios, como também os outliers, ou seja, aqueles que durante o exame não apresentaram nenhuma arritmia, ou então, apresentaram excessivas arritmias (isto é, que não podem ser atribuídas a estados emocionais). Quanto à situação ocupacional, entre os homens predominou a categoria "militar" (30\%), ao passo que entre as mulheres prevaleceu a categoria "do lar" (47\%). A seleção da amostra foi feita a partir da demanda espontânea de uma clínica cardiológica do Distrito Federal, obtendo-se a concordância dos participantes com a aplicação do Termo de Consentimento Livre e Esclarecido, tal como informado no projeto de pesquisa aprovado pelo Comitê de Ética da Faculdade de Medicina da Universidade de Brasília.

\section{Equipamento}

O aparelho de Holter é utilizado para avaliação de sintomas que podem ser causados por alteração do ritmo cardíaco e a duração de 24 horas é aquela que apresenta melhor relação custo-benefício. Cada um dos batimentos cardíacos é analisado conforme a sua morfologia, duração e precocidade. $\mathrm{O}$ equipamento, constituído por um gravador de registro, é preso à cintura da pessoa por meio de um cinto e ligado a eletrodos afixados ao tórax. Os dados de cada paciente são primeiramente transferidos para um cartão de memória, acoplado ao aparelho. As informações eletrocardiográficas gravadas são processadas por computador e os dados são transformados em gráficos e sintetizados em uma tabela, denominada de Relatório Tabular. Além deste registro, o exame de Holter é acompanhado por um Relatório de Atividades preenchido pelo próprio paciente (Grupi, Brito \& Uchida, 2001).

\section{Procedimento}

Como usualmente é feito, afixou-se o aparelho à cintura do participante, fornecendo-se as orientações necessárias. Solicitou-se o registro de todas as atividades realizadas durante o período de 24 horas, com indicação de início e término de cada atividade, bem como as emoções concomitantes. Caso emoções diferentes ocorressem durante uma mesma atividade, o paciente deveria registrar essa mudança emocional, sua intensidade e o horário. Esses registros constituíram o Relatório Diário. No dia seguinte, após a retirada do aparelho e avaliação pelo cardiologista, o paciente era entrevistado pela pesquisadora-psicóloga a partir de um roteiro previamente elaborado. O objetivo dessa entrevista era levantar informações complementares sobre as atividades diárias auto-registradas e esclarecer aspectos da história de vida. Os relatos foram gravados em áudio e, posteriormente, transcritos. O Relatório Diário de cada participante foi emparelhado hora a hora com os registros do Relatório Tabular, o qual fornece informações sobre as arritmias, conforme a localização (ventriculares e supraventriculares). Aqueles períodos que, mesmo após complementação por entrevista, permaneceram sem comentários foram registrados como "sem relato" (Sánchez, 2007). 


\section{Análise de dados}

Inicialmente, foram analisados os dados encontrados nos Relatórios Diários e Relatórios Tabulares por meio de estatística descritiva, utilizando-se a média aritmética dos registros. Cabe explicar que a análise foi focalizada no período de vigília, tendo sido excluídos relatos nos quais os pacientes afirmaram estar dormindo, visto que existem limitações relativas ao registro dessa experiência pelo próprio participante. Assim, para a estatística descritiva, os relatos emocionais foram organizados nas seguintes categorias e subcategorias: emoções positivas (abençoado, alegria, satisfação e segurança), emoções neutras (tranquilidade) e emoções negativas (ansiedade, preocupação, raiva, surpresa e tristeza). Posteriormente, utilizou-se o software Alceste Analyse de Lexèmes Concurrents dans les Énnoncés Simples d'un Texte - para análise de conteúdo das entrevistas. Vale explicitar que esse software organiza o conteúdo de um texto, determinando uma classificação descendente hierárquica entre as classes das palavras que foram agrupadas. Essas classes são apresentadas sob a forma de um dendrograma, que ilustra a relação entre elas, a força de suas relações e a representatividade de cada uma. A análise consiste, então, em identificar o sentido dessas classes para nomeá-las como categorias temáticas coerentes com o contexto global do texto (Ribeiro, 2000).

\section{Resultados e Discussão}

\section{Distribuição das arritmias ao longo do Exame de Holter $24 \mathrm{~h}$}

As mulheres apresentaram, em média, mais arritmias do que os homens, tanto ventriculares (respectivamente, 121,82 e 68,15) quanto supraventriculares (respectivamente, 265,59 e 67,69$)$. De modo geral, esses resultados não corroboram a literatura, que afirma a prevalência de doenças cardiovasculares no gênero masculino, apesar dos dados epidemiológicos, divulgados pelo Ministério da Saúde em 2003, apontarem que, no Brasil, o percentual de mulheres que falecem por problemas cardíacos é maior que o de homens.

Conforme ressaltado anteriormente, grande parte das pesquisas é conduzida com pessoas do sexo masculino, sendo este um fator de risco considerado como já estabelecido. Porém, a presente investigação, a exemplo de outros trabalhos, como o de Lima e Nussbacher (1996), alerta sobre a falta de atenção às diferenças entre os gêneros e, em especial, aos cuidados de saúde destinados à população feminina. É interessante comentar que as mulheres apresentaram mais que $o$ dobro de arritmias supraventriculares do que ventriculares, enquanto os homens apresentaram um número similar de ocorrências de arritmias.

Arritmias ventriculares. Conforme mostrado na Figura 1, a ocorrência de arritmias ventriculares apresentou uma distribuição distinta, entre mulheres e homens, ao longo do dia. No sexo feminino, verificou-se que entre 11:00 h e 12:00 h da manhã, houve o maior número de ocorrências de arritmias ventriculares, alcançando 400 , ou seja $19,3 \%$ dos registros $(\mathrm{N}=2.071)$. Esse é o horário em que as donas de casa estavam envolvidas com a preparação do almoço, conforme verbalizado em algumas entrevistas. A partir das 23:00 h até às 5:00 h, observou-se uma diminuição na quantidade de arritmias ventriculares, com um discreto aumento entre 2:00 h e 3:00 h, período em que é possível que estivessem ocorrendo sonhos. Já no sexo masculino, entre 22:00 h e 2:00 h ocorreu o maior número de arritmias ventriculares e, no período das 2:00 h às 11:00 h, o menor número do dia, embora tenha havido um leve aumento entre 3:00 h e 5:00 h.

Giannotti-Hallage (1990) diz que "os estados emocionais alterados parecem não precipitar episódios de arritmias instantaneamente, podendo ocorrer após períodos prolongados de preocupação, desapontamento, tensão emocional e insônia" (p.76). Dessa forma, é possível supor que, nos homens, a tensão acumulada ao longo do dia se manifestasse apenas

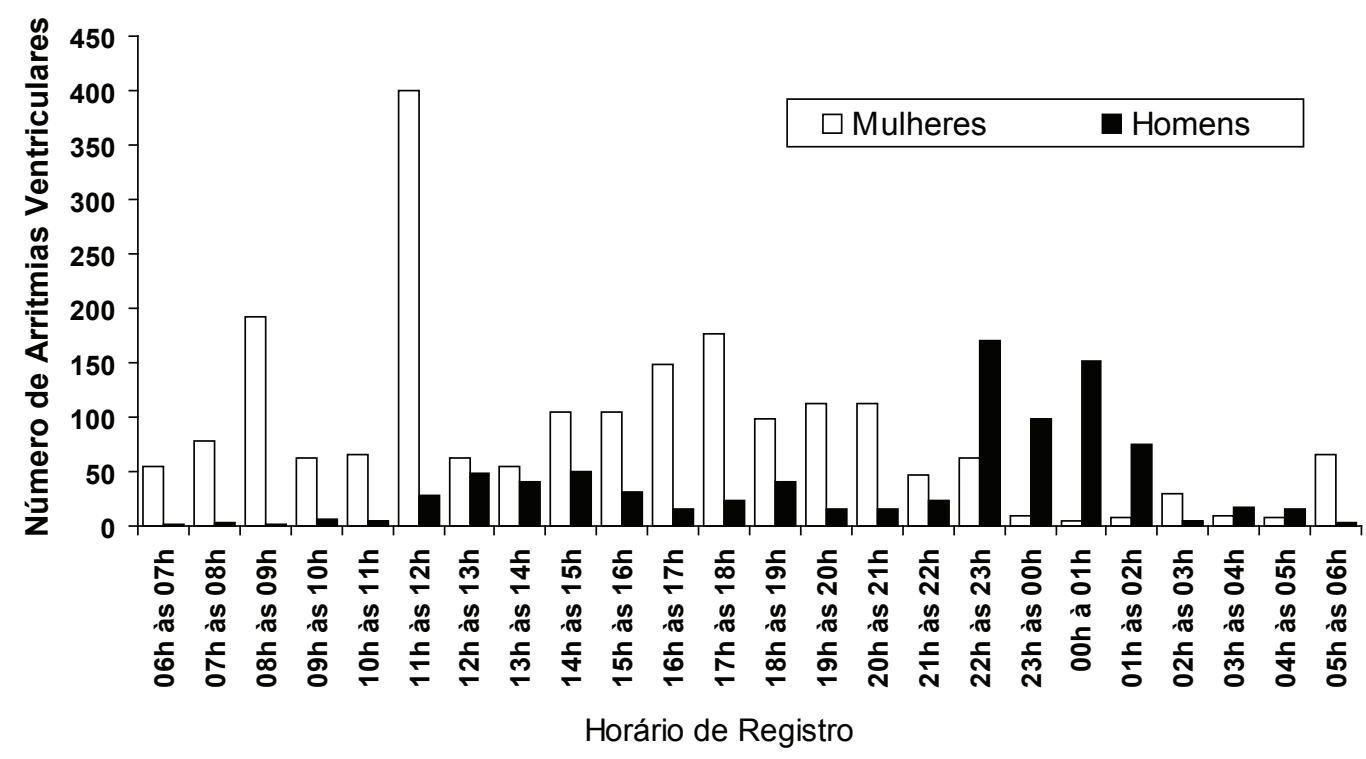

Figura 1. Distribuição das arritmias ventriculares ao longo do exame no sexo feminino $(\mathrm{N}=2.071)$ e no sexo masculino $(\mathrm{N}=886)$. 
durante a noite, o que não se identificou no conjunto de informações sobre as mulheres. Segundo Campos (1992), sonhos de forte conteúdo emocional acompanham reações cardiovasculares adrenérgicas, o que também pode contribuir para a ocorrência dessas arritmias.

Arritmias supraventriculares. A Figura 2 mostra que as arritmias supraventriculares estiveram mais concentradas no período vespertino e início do período noturno, tanto para as mulheres quanto para os homens. Mas, para as mulheres, os picos se deram mais cedo, entre 15:00 h e 18:00 h $(31,4 \%$ das ocorrências). Para os homens, os picos ocorreram no período compreendido entre 17:00 h e 19:00 h (22,7\% das ocorrências), o que coincide com os dados obtidos por Muller e cols. (1987, conforme citado por Bortolotto, 1999). O número de ocorrências aumentou no início da manhã para ambos os gêneros (a partir das 6:00 h para mulheres e a partir das 7:00 h para homens), talvez em função da liberação de cortisol que ocorre no despertar (Bortolotto, 1999).

\section{Distribuição das arritmias por categoria de emoção}

Verifica-se que homens e mulheres nomearam a mesma quantidade de subcategorias de emoções positivas (três cada), mas, por outro lado, as mulheres vivenciaram emoções negativas mais diferenciadas (ver Tabela 1). Constata-se, ainda, a variação desses registros entre os gêneros, sendo que a maior diferença ocorreu nas emoções positivas. A alegria foi a emoção mais percebida pelas mulheres. A emoção neutra, tranquilidade, foi a mais citada dentre o conjunto de emoções, principalmente entre os homens. Quanto às emoções negativas, os relatos de preocupação foram os únicos semelhantes entre os dois grupos. Ansiedade e tristeza assemelharam-se, sendo um pouco mais citadas pelas mulheres. Já a emoção raiva, foi mais mencionada pelos homens, o que corrobora a revisão de literatura reportada inicialmente, ao passo que surpresa não foi assinalada por eles e foi citada por apenas uma mulher. Vale comentar que esta pesquisa propõe uma classificação específica das emoções, visto que, conforme destacado anteriormente, as taxonomias adotadas pela literatura têm enfatizado, sobretudo, as emoções negativas (Fredrickson, 1998).

Emoções simultâneas a arritmias ventriculares. A simultaneidade entre as emoções e as arritmias revelou-se diferente em homens e mulheres (ver Figura 3). Com relação às emoções positivas, a subcategoria mais associada a arritmias ventriculares, por ambos os gêneros, foi sentir-se abençoado. No entanto, esta subcategoria teve um número pequeno de registros. É importante acrescentar que entre esses registros, um homem e duas mulheres relataram essa emoção ao rezar. A relação entre saúde e religiosidade ainda não está claramente estabelecida na literatura, mas estudos mostram associação positiva entre níveis de envolvimento religioso e indicadores de bem estar psicológico, como satisfação de vida. Nesse sentido, o impacto positivo do envolvimento religioso parece ser maior em indivíduos com mais idade e pessoas com deficiências e doenças, havendo diferenças estatisticamente significativas entre os gêneros em relação ao uso desta estratégia de enfrentamento (Moreira-Almeida, Lotufo Neto \& Koenig, 2006).

De acordo com os dados da Tabela 1, a alegria foi a segunda emoção mais relatada pelas mulheres. Essa emoção foi vivenciada por elas principalmente em situações de atividades com familiares, com amigos e assistindo televisão. Já para os homens, a alegria representou apenas 2,2\% dos registros e, em todos os casos, ela estava relacionada com atividades com amigos. Contudo, como indicado na Figura 3 , para mulheres e homens, a alegria poucas vezes ocorreu simultaneamente com arritmias ventriculares. Chama a atenção que segurança não foi mencionada por mulheres e os homens não identificaram satisfação. Conforme salientado antes, são necessárias mais pesquisas para compreender tais evidências sobre emoções positivas, pois a ênfase da literatura tem sido para as emoções negativas, o que acaba por nortear orientações de atendimento simplistas e redutoras

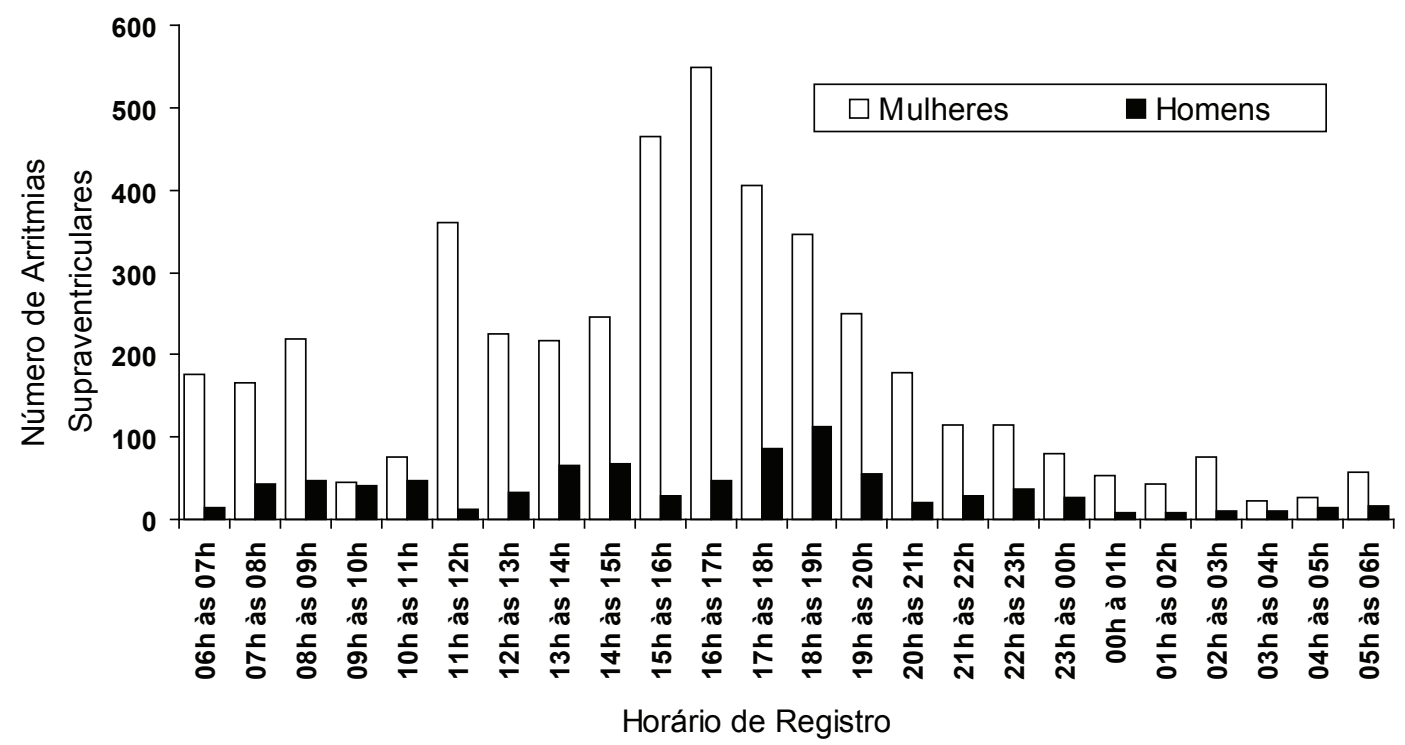

Figura 2. Distribuição das arritmias supraventriculares ao longo do exame no sexo feminino $(\mathrm{N}=4.515)$ e no sexo masculino $(\mathrm{N}=880)$. 
Tabela 1. Total de registros de cada estado emocional relatado pelos participantes.

\begin{tabular}{|c|c|c|c|c|c|}
\hline \multirow{2}{*}{ Categorias } & \multirow{2}{*}{ Subcategorias } & \multicolumn{2}{|c|}{ MULHERES } & \multicolumn{2}{|c|}{ HOMENS } \\
\hline & & Total & $\%$ & Total & $\%$ \\
\hline \multirow{4}{*}{ Positiva } & Abençoado & 5 & 3,1 & 1 & 0,7 \\
\hline & Alegria & 27 & 17,0 & 3 & 2,2 \\
\hline & Satisfação & 8 & 5,0 & - & - \\
\hline & Segurança & - & - & 3 & 2,2 \\
\hline \multirow[t]{3}{*}{ Neutra } & Tranquilidade & 68 & 42,8 & 81 & 60,5 \\
\hline & Ansiedade & 14 & 8,8 & 9 & 6,7 \\
\hline & Preocupação & 16 & 10,1 & 17 & 12,7 \\
\hline \multirow[t]{3}{*}{ Negativa } & Raiva & 9 & 5,7 & 12 & 9,0 \\
\hline & Surpresa & 1 & 0,6 & - & - \\
\hline & Tristeza & 11 & 6,9 & 8 & 6,0 \\
\hline Total & & 159 & 100 & 134 & 100 \\
\hline
\end{tabular}

de que as emoções, como um todo, são prejudiciais à saúde (Maciel, 1994).

Ainda de acordo com a Figura 3, a emoção neutra, tranquilidade, acompanhou arritmias ventriculares mais frequentemente entre as mulheres do que entre os homens. Em termos de emoções negativas, nas mulheres, a preocupação relacionou-se mais com arritmias ventriculares do que a ansiedade e nos homens, essa relação foi inversa. A raiva mostrou-se relacionada a arritmias ventriculares nos homens, mas não nas mulheres; e a tristeza não foi simultânea a arritmias ventriculares em nenhum dos gêneros, verificando-se o mesmo com a surpresa.
Emoç̃̃es simultâneas a arritmias supraventriculares. Conforme mostrado na Figura 4, à exceção de segurança, as emoções positivas abençoado, alegria e satisfação ocorreram mais entre as mulheres do que entre os homens. $\mathrm{Na}$ subcategoria tranquilidade, as arritmias supraventriculares registraram-se da seguinte maneira: média 7,0 para as mulheres e 5,0 para os homens.

A ansiedade é a única emoção que está mais relacionada a arritmias supraventriculares em homens do que em mulheres; no entanto, a diferença é pequena (média 1,9 e 1,3 respectivamente). As demais emoções negativas (preocupação, raiva e tristeza) foram registradas com maior frequência entre as

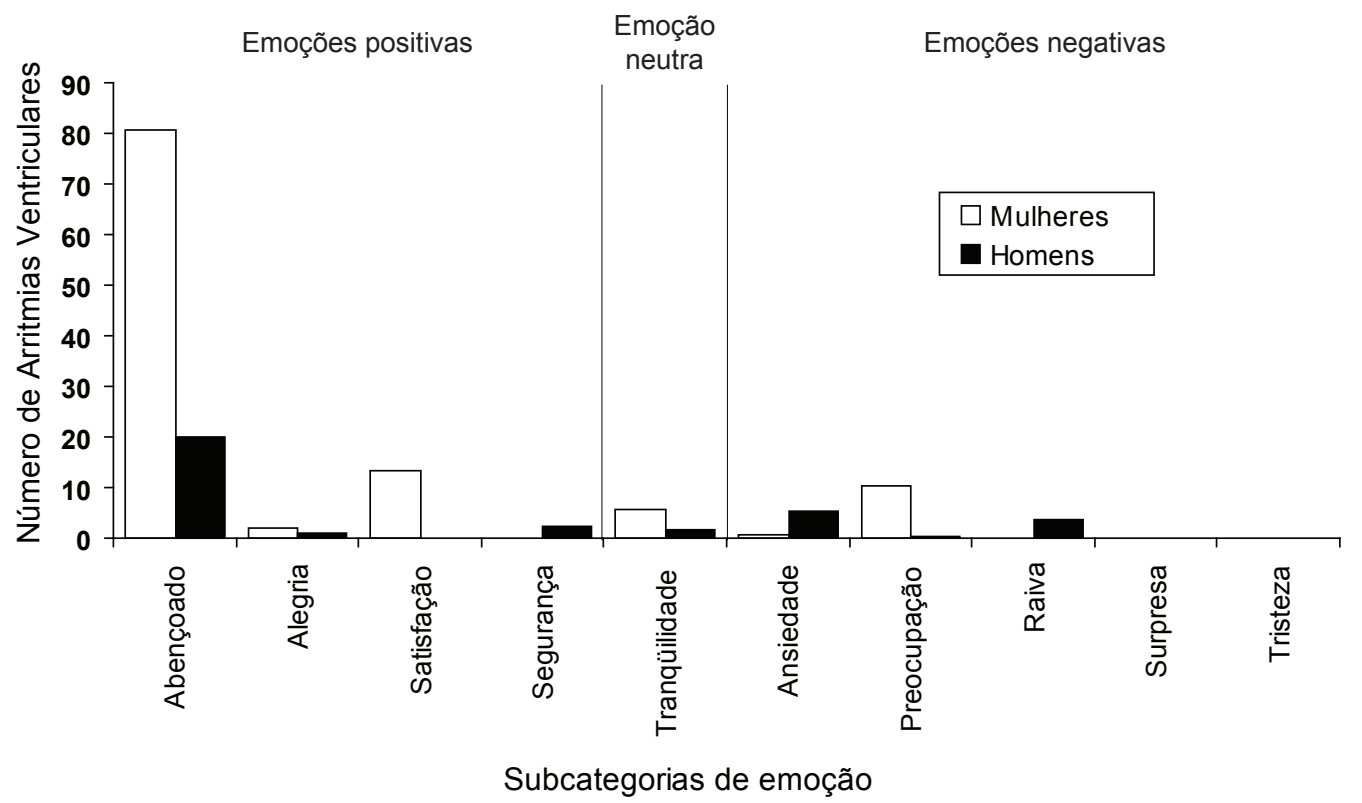

Figura 3. Média de arritmias ventriculares em cada subcategoria de emoções relatadas 


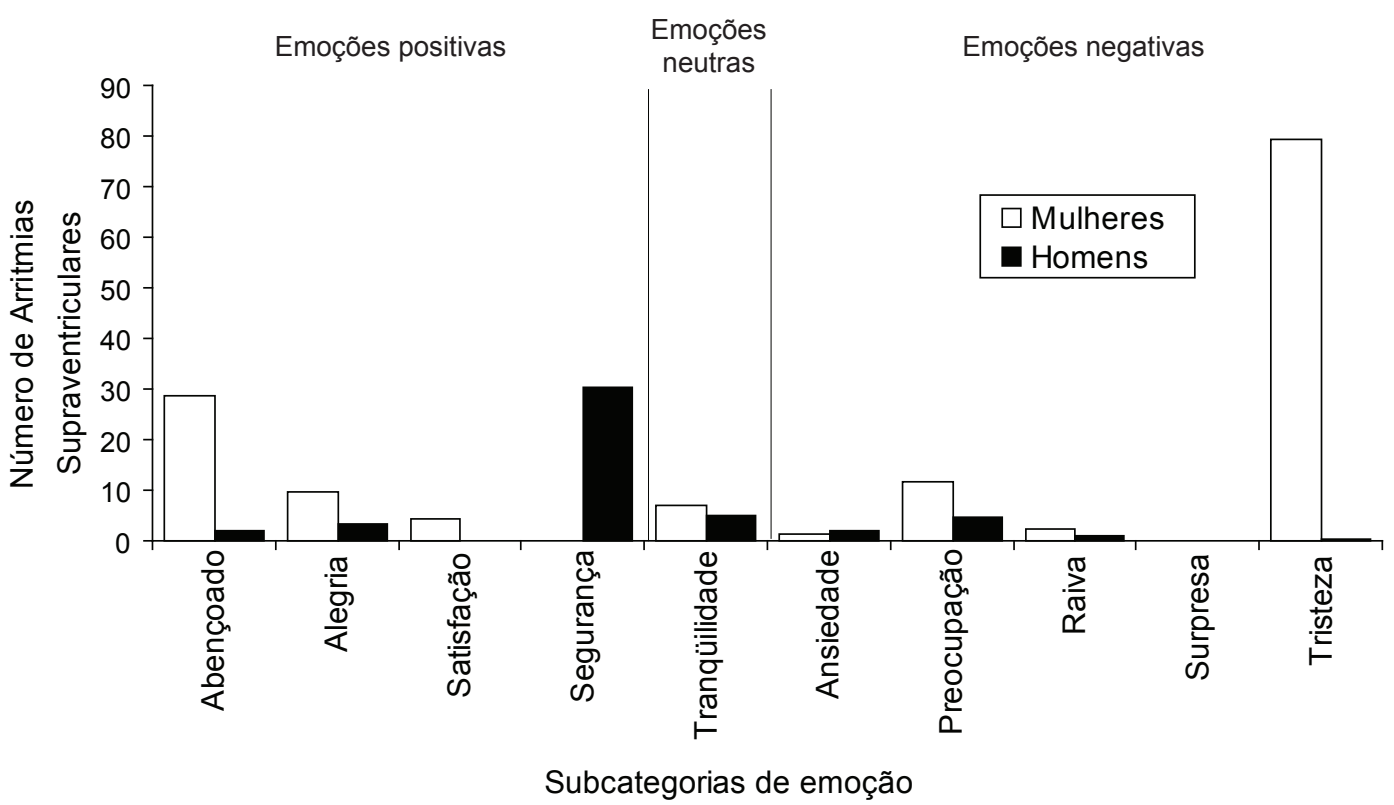

Figura 4. Média de arritmias supraventriculares em cada subcategoria de emoções relatadas

mulheres. É interessante ressaltar, ainda, a média de 79,3 arritmias supraventriculares nos períodos em que as mulheres também relataram tristeza.

Comparação entre emoções simultâneas a arritmias ventriculares e supraventriculares. A maioria das emoções relatadas pelos participantes apresentou-se mais simultânea a arritmias supraventriculares do que ventriculares (ver Figuras 3 e 4). Destaca-se nessa comparação que a média de registros de tristeza concomitantes a arritmias ventriculares foi baixa: 0,1 para ambos os gêneros. $O$ mesmo não foi verificado para as supraventriculares, pois essa média foi a mais alta entre as mulheres e muito baixa entre os homens: 79,3 e 0,5 , respectivamente. A emoção raiva se diferenciou das demais emoções, pois foi simultânea a mais arritmias supraventriculares nas mulheres e ventriculares nos homens, o que confirma diferenças entre gêneros.

\section{Entrevistas}

O Diagrama 1 ilustra a relação das categorias de relato verbal, classificadas pelo software Alceste (Ribeiro, 2000). A categoria Estresse nas Relações Interpessoais reúne relatos de sofrimento decorrente da interação com pessoas significativas (como filhos e netos). Ou seja, existe a necessidade de reconhecimento pelo esforço realizado em prol dos outros. Quando esse reconhecimento não existe, ocorre frustração. A categoria Relações Interpessoais Rotineiras se opõe à anterior, pois reflete aspectos do cotidiano e da relação com pessoas próximas, mas que não trazem conflitos. A categoria Vivências do "Estar Doente" agrupa relatos sobre a dificuldade em assumir o papel de doente. A doença parece não fazer parte de si, é algo externo, apesar dos sintomas. Quando reconhecida, é atribuída a fatores externos. Na categoria Expressões das Vivências Emocionais, estão agrupados relatos referentes à dificuldade dos participantes em nomear o que sentem e identificar o que ocorre. $\mathrm{O}$ emocional é reconhecido como parte do problema, mas essa atribuição é difícil de ser compreendida por eles. A categoria Comportamentos, Saúde e Emoções é pouco associada às situações relacionais. Muitas vezes, os participantes manifestam o pensamento mágico de que as alterações orgânicas se dissiparão se deixarem de realizar determinadas ações. Esses resultados reafirmam o trabalho de Campos (1992) sobre aspectos psicossomáticos em Cardiologia e complementam a análise das arritmias ao longo do exame de Holter 24 horas.

\section{Considerações Finais}

Esta pesquisa verificou que o Exame de Holter 24 horas viabiliza identificar a simultaneidade entre emoções, experiência de vida e arritmias cardíacas em condições não laboratoriais. Constataram-se diferenças acentuadas entre gêneros: mulheres apresentaram mais arritmias que homens. Nas mulheres, arritmias ventriculares ocorreram mais simultaneamente à preocupação do que à ansiedade e não se mostraram relacionadas à raiva. Além disso, essa emoção se diferenciou das demais, pois foi simultânea a mais arritmias supraventriculares nas mulheres e ventriculares nos homens. A tristeza não foi associada a arritmias ventriculares por nenhum dos gêneros, mas foi relacionada ao maior número de arritmias supraventriculares entre as mulheres.

Considera-se que, se o conhecimento sobre a participação das emoções na arritmogênese for continuamente aprimorado, será possível gerar subsídios para uma orientação psicológica mais adaptada à rotina diária dos pacientes. Em suma, compreender como os fatores emocionais desencadeiam o desequilíbrio funcional do coração pode fundamentar estratégias terapêuticas mais eficazes em Cardiologia. 


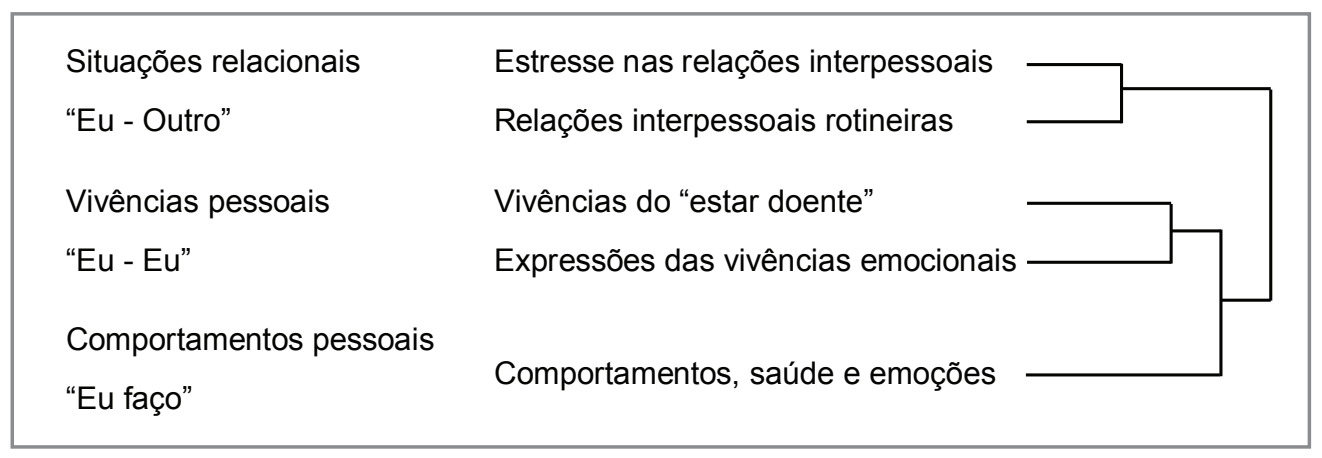

Diagrama 1. Categorias temáticas das entrevistas segundo a classificação hierárquica descendente.

\section{Referências}

Almeida, O. P., \& Fráguas Jr., R. (1996). Depressão e doença cardiovascular. Revista da Sociedade de Cardiologia do Estado de São Paulo, 6, 749-756.

Bortolotto, L. A. (1999). Implicações clínicas dos ritmos biológicos do sistema cardiovascular - Parte 2: cronobiologia dos eventos cardiovasculares. Revista Brasileira de Cardiologia, 1, 160164.

Burg, M. M., Lampert, R., Joska, T., Batsford, W., \& Jain, D. (2004). Psychological traits and emotion-triggering of ICD shock-terminated arrhythmias. Psychosomatic Medicine, 66, 898-902.

Campos, E. P. (1992). Aspectos psicossomáticos em cardiologia. Em J. M. Filho (Org.), Psicossomática hoje (pp. 234- 252). Porto Alegre: Artes Médicas.

Carels, R. A., Sherwood, A., Babyak, M., Gullette, E. C., Coleman, R. E., Waugh, R., Jiang, W., \& Blumenthal, J. A. (1999). Emotional responsivity and transient myocardial ischemia. Journal of Consulting and Clinical Psychology, 67, 605-610.

Chang, P. P., Ford, D. E., Meoni, L. A., Wang, N. Y., \& Klag, M. J. (2002). Anger in young men and subsequent premature cardiovascular disease: The precursors study. Archives of Internal Medicine, 168, 901-906.

Danner, D. D., Snowden, D. A., \& Friesen, W. V. (2001). Positive emotions in early life and longevity findings from the nun study. Journal of Personality and Social Psychology, 80, 804-813.

Eaker, E. D. (1998). Psychosocial risk factors for coronary heart disease in women. Cardiology Clinics, 16, 103-111.

Favarato, M. E. C. S., \& Aldrighi, J. M. (2001). A mulher coronariopata no climatério após a menopausa: implicações na qualidade de vida. Revista da Associação Médica Brasileira, 47, 339-345.

Frasure-Smith, N., \& Lesperance, F. (1998). Role of psycho-social factors in CVD. Evidence-Based Cardiovascular Medicine, 2, 64-65.

Fredrickson, B. L. (1998). What good are positive emotions?. Review of General Psychology, 2, 300-319.

Gallo, L. C., \& Matthews, K. A. (2003). Understanding the association between socioeconomic status and physical health: Do negative emotions play a role? Psychological Bulletin, 129, 10-51.

Giannotti-Hallage, A. (1990). Papel das emoções e das situações psicologicamente estressantes na gênese das arritmias cardíacas e morte súbita. Em B. W. R. Lamosa (Org.), Psicologia aplicada à Cardiologia. (pp 71-78). São Paulo: Fundo Editorial Byk.
Grupi, C. J., Brito, F. S., \& Uchida, A. H. (2001). Eletrocardiograma de longa duração: o sistema Holter. Em P. J. Moffa \& P. C. R. Sanches (Orgs.), Eletrocardiograma normal e patológico (pp. 801-838). São Paulo: Roca.

Hupka, R. B., Lenton, A. P., \& Hutchison, K. A. (1999). Universal development of emotion categories in natural language. Journal of Personality and Social Psychology, 77, 247-278.

Kubzansky, L. D., \& Kawachi, I. (2000). Going to the heart of the matter: Do negative emotions cause coronary heart disease? Journal of Psychosomatic Research, 48, 323-337.

Labouvie-Vief, G., Lumley, M. A., Jain, E., \& Heinze, H. (2003). Age and gender differences in cardiac reactivity and subjective emotion responses to emotional autobiographical memories. Emotion, 3, 115-126.

Lampert, R., Joska, T., Burg, M. M., Batsford, W. P., McPherson, C. A., \& Jain, D. (2002). Emotional and physical precipitants of ventricular arrhythmia. Circulation, 106, 1800-1805.

Lavoiea, K. L., Miller, S. B., Conwaya, M., \& Fleetb, R. P. (2001). Anger, negative emotions, and cardiovascular reactivity during interpersonal conflict in women. Journal of Psychosomatic Research, 51, 503-512.

Lima, J. A. C., \& Nussbacher, A. (1996). O coração da mulher é diferente?. Revista da Sociedade de Cardiologia do Estado de São Paulo, 6, 704-706.

Lipp, M. E. N., Pereira, M. M. B., Justo, A. P., \& Matos, T. M. G. (2006). Cardiovascular reactivity in hypertensives: Differential effect of expressing and inhibiting emotions during moments of interpersonal stress. Spanish Journal of Psychology, 9, 154-161.

Maciel, C. L. C. (1994). Emoção, doença e cultura: O caso da hipertensão essencial. Em B. W. Romano (Org.), A prática da psicologia nos hospitais (pp. 1-38). São Paulo: Pioneira.

Ministério da Saúde (2003). Estatísticas vitais: mortalidade e nascidos vivos. Retirado em 07/03/2006 de http://tabnet.datasus. gov.br/cgi/tabcgi.exe?sim/cnv/obtuf.def

Moreira-Almeida, A., Lotufo Neto, F., \& Koenig, H. G. (2006). Religiousness and mental health: A review. Revista Brasileira de Psiquiatria, 28, 242-250.

Neubern, M. S. (2000). As emoções como caminho para uma epistemologia complexa da psicologia. Psicologia: Teoria e Pesquisa, 16, 153-164.

Perez, G. H. (2004). Fatores de risco da doença arterial coronária em mulheres: uma visão psicossomática. Em A. L. A. Ribeiro \& D. P. Rosa (Orgs.), Mulher \& coração: aspectos psicológicos ligados à cardiopatia (pp. 89-104). São Paulo: Papirus. 
Perez, G. H., Nicolau, J. C., Romano, B. W., \& Laranjeira, R. (2005). Depressão e síndromes isquêmicas miocárdicas instáveis: diferenças entre homens e mulheres. Arquivos Brasileiros de Cardiologia, 85, 319-326.

Pinton, F. A., Carvalho, C. F., Miyazaki, M. C. O. S., \& Godoy, M. F. (2006). Depressão como fator de risco de morbidade imediata e tardia pós-revascularização cirúrgica do miocárdio. Brazilian Journal of Cardiovascular Surgery, 21, 68-74.

Ribeiro, A. S. M. (2000). Alceste: análise quantitativa de dados textuais. Manual não publicado, Universidade de Brasília, Brasília.

Richman, L. S., Kubzansky, L., Maselko, J., Kawachi, I., Choo, P., \& Bauer, M. (2005). Positive emotion and health: Going beyond the negative. Health Psychology, 24, 422-429.

Rozanski, A., Blumenthal, J. A., \& Kaplan, J. (1999). Impact of psychological factors on the pathogenesis of cardiovascular disease and implications for therapy. Circulation, 99, 21922217.
Sánchez, A. M. (2007). Abordagem psicológica das arritmias cardiacas: uma análise das emoções relatadas em exame de Holter. Dissertação de Mestrado, Universidade de Brasília, Brasília.

Sirois, B. C., \& Burg, M.M. (2003). Negative emotion and coronary heart disease. Behavior Modification, 27, 83-102.

Swan, G. E., \& Carmelli, D. (1996). Curiosity and mortality in aging adults. Psychology and Aging, 11, 449-453.

Thomas, D. L., \& Diener, E. (1990). Memory accuracy in the recall of emotions. Journal of Personality and Social Psychology, 59, 291-297.

Recebido em 18.12.07

Primeira decisão editorial em 17.06.08

Versão final em 20.06.08

Aceito em 17.09.08 